\title{
Toroidal black holes and topological censorship
}

\author{
Stuart L. Shapiro \\ Center for Astrophysics and Relativity, 326 Siena Drive, Ithaca, New York 14850 \\ Saul A. Teukolsky \\ Center for Radiophysics and Space Research, Cornell University, Ithaca, New York 14853 \\ Jeffrey Winicour \\ Department of Physics and Astronomy, University of Pittsburgh, Pittsburgh, Pennsylvania 15260
}

(Received 21 July 1995)

\begin{abstract}
Recent theorems regarding topological censorship are in apparent conflict with simulations of the collapse of rotating matter to form toroidal black holes. The geometry of a temporarily toroidal event horizon is analyzed and shown to be completely consistent with the theorems. A simple flat space model provides insight into the geometry of a toroidal black hole.
\end{abstract}

PACS number(s): 04.70.Bw, 02.40.-k, 04.20.Gz, 04.25.Dm

\section{INTRODUCTION}

There are several theorems which state that under various conditions a black hole must have spherical topology. On the other hand, recent computer simulations of collapsing matter in a fully general relativistic setting have given rise to black holes that initially form with toroidal topology and only at a later time become spherical. In this paper we show that the computational results are in fact consistent with the theorems and can be understood in terms of a simple spacetime model of a toroidal event horizon.

The first results concerning the spherical topology of black holes came from the analysis of exact solutions for the time independent Schwarzschild and Kerr spacetimes. The global geometry of these spacetimes revealed that they possess an event horizon, which forms the boundary of the region visible by distant observers. This horizon is a three-dimensional null hypersurface whose null generators extend for infinite affine length both in the past and the future. Topologically, the Schwarzschild and Kerr horizons are the product of a line and a sphere. The line represents a null generator. The surface of a Kerr or Schwarzschild black hole corresponds to the intersection of the horizon with a spacelike hypersurface. This is the sphere.

Subsequently, the definitions required to describe black holes were refined with a high degree of technical precision. This led the way for more general theorems regarding black hole properties. The concept of distant observers is formulated rigorously in terms of future null infinity $\mathcal{I}^{+}$, as constructed in the Penrose compactification of an asymptotically flat spacetime [1]. The event horizon $\mathcal{H}^{+}$is defined as the boundary of the causal past of $\mathcal{I}^{+}$. Here the causal past of a region $\Sigma$ is formally denoted by $J^{-}(\Sigma)$ and consists of the points that can be connected to $\Sigma$ by a future directed timelike or null curve. In terms of this formal notation, $\mathcal{H}^{+}=\dot{J}^{-}\left(\mathcal{I}^{+}\right)$. General results concerning causal boundaries imply that
$\mathcal{H}^{+}$is generated by null geodesics [2]. Moving along the future direction, null generators can enter the horizon but existing generators cannot leave. The black hole region consists of those spacetime points not contained in $J^{-}\left(\mathcal{I}^{+}\right)$, with the event horizon on its boundary [3]. A black hole at a particular instant of time is defined by a connected component of the intersection of the black hole region with a Cauchy hypersurface. (A Cauchy hypersurface is a spacelike hypersurface with global extent sufficient to specify a unique evolution by means of initial Cauchy data.) At the time defined by this hypersurface, this intersection may consist of several disjoint regions, in which case there would exist the corresponding number of black holes at that time.

The surface topology of a black hole at a given time is the topology of the intersection of the Cauchy hypersurface (defining the time) with the event horizon. In the Schwarzschild and Kerr vacuum spacetimes, this topology is spherical at all times. The same is true in the nonstationary case of an Oppenheimer-Snyder spacetime, in which the event horizon is formed by the collapse of a spherical ball of matter. It is evident that a spherically symmetric black hole must have spherical surface topology. Consequently, it should not be surprising that small deviations from spherical symmetry or small perturbations of the Kerr spacetime preserve the spherical black hole topology. A number of theorems have established much stronger results.

The simplest situation arises in the general stationary spacetime, for which it can be shown that any black hole must have the surface topology of a two-sphere [5]. The first theorem regarding the topology of nonstationary black holes is due to Gannon [6]. Assuming a physically reasonable condition of asymptotic flatness, he proved that the surface topology of a smooth black hole must be either a two-sphere or a torus (provided that the energymomentum tensor of the matter fields satisfies the dominant energy condition).

Gannon's approach has recently been extended and 
generalized to yield stronger theorems, under the assumptions of asymptotic flatness, global hyperbolicity, and a suitable energy condition on the matter fields. Under the title of "topological censorship," Friedmann, Schleich, and Witt proved a theorem that any two causal curves extending from past to future null infinity may be deformed into each other (in the sense of homotopy) [7]. As Jacobson and Venkataramani have pointed out, a black hole with toroidal surface topology provides a potential mechanism for violating topological censorship by sending a light ray from the infinite past through the hole in the torus and back out to future null infinity [8]. This light ray would not be deformable to a light ray that skirts the horizon altogether. Thus the topological censorship theorem implies that the hole in a toroidal horizon must close up quickly, before a light ray can pass through. Jacobson and Venkataramani have also established a theorem that strengthens a recent result due to Browdy and Galloway that the surface geometry of a black hole at a given time must be a two-sphere if no new null generators enter the horizon at later times [9]. The theorem of Jacobson and Venkataramani limits the time for which a toroidal black hole can persist, albeit in a highly technical way.

It is the resolution of the potential paradox, raised by the discovery of toroidal black holes in the computational simulation of the gravitational collapse of rotating matter [10], that has motivated our investigation. The computer code is designed to evolve rotating axisymmetric spacetimes in full general relativity [11]. The source of the gravitational field in the simulations is a swarm of collisionless matter particles. The code can follow the collapse of a rotating collisionless cluster to a Kerr black hole.

The code has been subjected to a number of tests to assure that it is accurate. Among these tests are the propagation of linearized gravitational waves of both polarizations, for which analytic solutions exist; maintaining rotating equilibrium configurations in stable equilibrium for many dynamical timescales; and verifying that the black holes that form from collapse settle down to Kerr holes at late times. In addition, during the course of numerical evolution, we compute a set of physical diagnostics to monitor the code's reliability in the nonlinear regime. We compute the Brill mass and angular momentum of the spatial hypersurfaces and look for trapped regions. The Brill mass, corrected for the loss of gravitational radiation through the outer boundary, is a conserved quantity. Since in axisymmetry gravitational radiation carries no angular momentum, the total angular momentum of the system is another conserved quantity. We locate the apparent horizon during black hole formation and probe its geometry. Once the evolution is completed and the black hole settles down, we map out the event horizon and ergoregion and probe their geometries. Although we cannot prove mathematical theorems by means of a numerical simulation, our tests give us confidence that the results reported in Ref. [11] are correct.

The plan of this paper is as follows. In Sec. II we present a simple flat space model of a horizon that has toroidal topology. This provides insight into the visualization of the spacetime geometry of a toroidal horizon. However, because the model has the property of structural stability, it also provides important clues for the case of gravitational collapse. Indeed, in Sec. III we analyze the spacetime geometry of the horizon formed in the computational simulation of the collapse of a rotating toroid of collisionless particles. The qualitative structure is in agreement with the flat space model when the deformation induced by curvature is taken into account. Finally, we show that our results are in complete accord with the above black hole theorems.

\section{THE HORIZON OF AN OBLATE SPHEROID}

Given any two-dimensional surface of spherical topology $\Sigma$ we can construct a model event horizon in flat spacetime by considering $\dot{J}^{-}(\Sigma)$, which is the boundary of the spacetime points in the causal past of $\Sigma$. It will be generated by past-directed light rays emanating normal to $\Sigma$. In general, $\dot{J}^{-}(\Sigma)$ consists of an inner and an outer component, corresponding to the inward and outward pointing normals to $\Sigma$. Here we concentrate only on the inner component. If $\Sigma$ were a "surface at infinity," there would be no outer component. A light ray $L_{1}$ generating $\dot{J}^{-}(\Sigma)$ continues backward in time unless it "meets" another generator. This can happen either by crossing another generator $L_{2}$ that emanates from a separate point of $\Sigma$, or by focusing to a point along with a neighboring set of generators (in the mathematical sense of a vanishing Jacobi field). The corresponding crossover points and caustic points are where $\dot{J}^{-}(\Sigma)$ originates, as follows from the elementary properties of causal sets [2]. Furthermore, the crossover points will in general form a spacelike two-dimensional surface. This is analogous to the way that the null hyperplane $z=t$ intersects the null hyperplane $z=-t$ in the $(x, y)$ plane (at $t=0)$. (We take the velocity of light equal to unity.)

The case in which $\Sigma$ is an oblate spheroid is of particular relevance to the issue of toroidal horizons. As a visualization aid, first consider the spatial picture of light rays streaming inward from an initially oblate, spheroidal wave front, as illustrated in Fig. 1. We have chosen the $z$ axis to be the rotational symmetry axis of the spheroid. The figure is in the $(x, z)$ plane but this supplies all the information about the geometric optics because the wave front is axially symmetric. Each pair of opposing points at $(+x, z)$ and $(-x, z)$ corresponds to a circle of symmetry in the $(x, y)$ plane (except for points on the axis). The figure is also reflection symmetric about $z=0$, so it is sufficient to indicate just the rays emanating (backward in time) from the $z>0$ half of the spheroid. These rays all intersect rays emanating from the $z<0$ half at the crossover points $X$, at $z=0$, before they caustic. The limiting rays, traveling along the $x$ axis, focus at the boundary $C$ of the crossover $X$. The caustics of the rays starting with $z>0$ are also shown, but these are encountered after crossing $X$ so they are in the past of the 


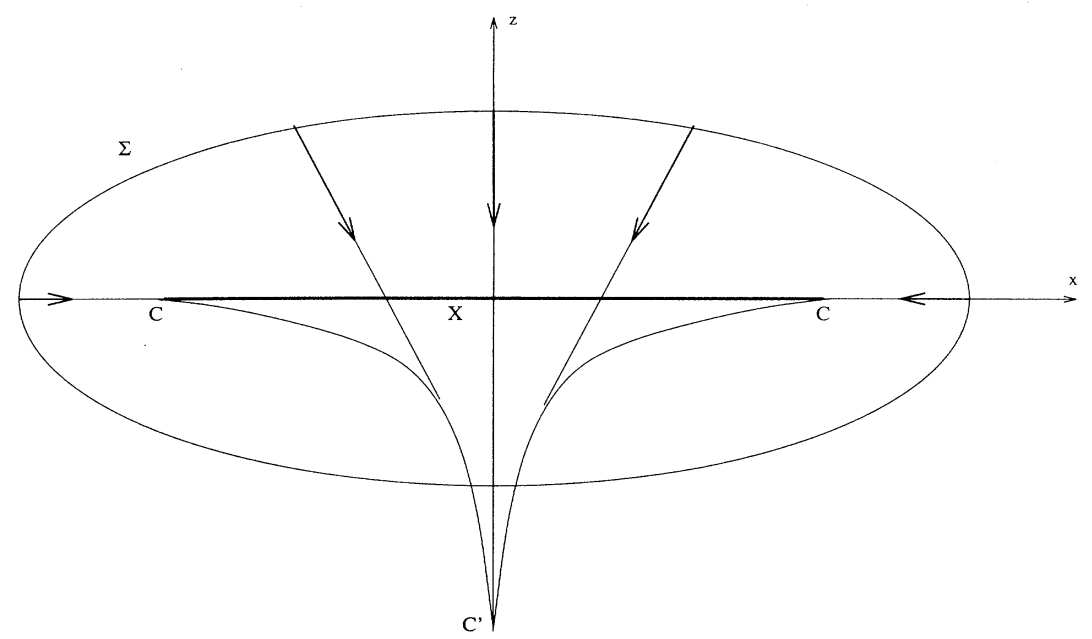

FIG. 1. Spatial picture of the cusps $C$ and $C^{\prime}$, connected by fold lines, for the rays emanating from a spheroidal wave front $\Sigma$. Opposing rays intersect in the (thicker shaded) crossover line $X$.

horizon associated with this wave front. So the horizon is generated by rays that begin at $X$ and its boundary $C$. Taking into account the $y$ dependence, $X$ is a disc bounded by a caustic ring $C$.

Now consider the corresponding spacetime picture. Since $X$ is a crossover surface it must be spacelike. However, as it approaches the caustic ring $C$ an interesting feature develops. The distance from the spheroidal wave front to $X$, measured along the rays, is longest at the center and decreases toward the edge at $C$. Consequently, the rays traced backward in time from the wave front reach $X$ at different times. Figure 2 gives a spacetime version of Fig. 1. The following analysis shows that $X$ becomes asymptotically lightlike at $C$.

In order to describe the mathematical details underlying the geometrical optics, let the profile of a spheroidal wave front $\Sigma$ in the $(x, z)$ plane be given in terms of a parameter $\lambda$ by the ellipse

$$
x_{0}^{a}(\lambda)=\left[x_{0}(\lambda), z_{0}(\lambda)\right]=[\sin \lambda, a \cos \lambda] .
$$

We are concerned with the oblate case for which $a<1$.
The past-directed light rays traveling normally inward to the wavefront have paths $x^{a}(s, \lambda)=x_{0}^{a}(\lambda)+s N^{a}$, where $s$ is the distance measured along the rays and

$$
N^{a}=-\left(\sin \lambda, a^{-1} \cos \lambda\right) /\left(\sin ^{2} \lambda+a^{-2} \cos ^{2} \lambda\right)^{1 / 2}
$$

is the unit normal.

The Jacobi field $J^{a}=\partial x^{a} / \partial \lambda$ represents a vector field connecting neighboring rays. Caustics occur when the Jacobi field either vanishes or becomes tangent to the rays. Thus the necessary and sufficient condition for a caustic is that $J^{x} N^{z}-J^{z} N^{x}=0$, which leads to

$$
s=a^{2}\left(\sin ^{2} \lambda+a^{-2} \cos ^{2} \lambda\right)^{3 / 2} .
$$

This equation describes how the path length from the wave front to the caustic varies from $a^{2}$ (for a ray tangent to the equator) to $1 / a$ (for a ray along the $z$ axis). On the other hand, the path length from the wave front to the crossover point with coordinates $(x, 0)$ in the equatorial plane is given by
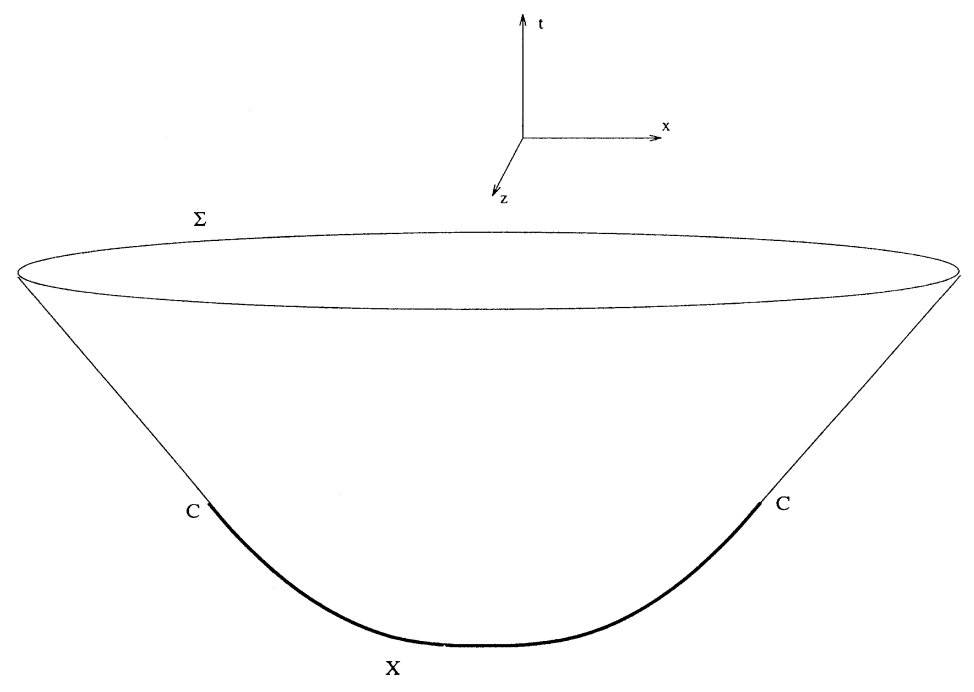

FIG. 2. Spacetime picture of the horizon formed by the wave front of Fig. 1. 


$$
s=a^{2}\left(\sin ^{2} \lambda+a^{-2} \cos ^{2} \lambda\right)^{1 / 2}=a\left[1+x^{2} /\left(a^{2}-1\right)\right]^{1 / 2},
$$

which varies from $a^{2}$ (for a ray tangent to the equator) to $a$ (for a ray along the $z$ axis). Thus all but the equatorial rays cross the equatorial plane between $a^{2}-1<x<$ $1-a^{2}$ before they focus.

With the velocity of light set to unity, $s$ gives the time at which the crossover points are reached, assuming that they all leave the initial wave front at $t=0$. Thus, from a spacetime point of view, (4) implies that $X$ is the surface of revolution generated by the spacetime curve

$$
t=-a\left[1+x^{2} /\left(a^{2}-1\right)\right]^{1 / 2} .
$$

Therefore, along $X$,

$$
\begin{aligned}
\frac{d t}{d x} & =\frac{a x}{\left(1-a^{2}\right) \sqrt{1+x^{2} /\left(a^{2}-1\right)}} \\
& =\frac{a \sin \lambda}{\sqrt{\cos ^{2} \lambda+a^{2} \sin ^{2} \lambda}},
\end{aligned}
$$

so that $d t / d x \rightarrow 1$ at the end points of $X$.

Thus $X$ becomes asymptotically lightlike as it approaches $C$, as illustrated in Fig. 2. The light rays originating on the surface $X$ and extending to the wave front $\Sigma$ generate a three-dimensional surface $H$ in a rough analogy to how an event horizon is formed in gravitational collapse. However, Fig. 2 is a flat spacetime picture that does not include the gravitational effect of curvature. In a precise analogy with an event horizon, $\Sigma$ should represent the final equilibrium state reached after the black hole has lost its "hair" and has stopped growing. In the rotating case this is expected to result in a final Kerr black hole with intrinsically oblate surface geometry. So, to make our analogy relevant to a rotating black hole, we should regard $\Sigma$ as a late time cross section of the horizon in a curved spacetime.

Spacetime curvature will deform the features shown in Fig. 2 by focusing the light rays traced back from $\Sigma$. However, the points $C$ are cusps, which are structurally stable caustics. In fact, cusps and their fold lines (the caustic curves $C C^{\prime}$ depicted in Fig. 1) are the unique stable caustics that occur in the presence of rotational symmetry [12]. Structural stability implies that, in a neighborhood of a cusp, the qualitative features of Fig. 2 should be unaffected by perturbations, whether introduced by the gravitational bending of light or by numerical error. A similar situation arises in the event horizon produced in the head-on merger of two black holes, where rotational symmetry also gives rise to cusps [13]. In that case, focusing effects cause the crossover line (analogous to $X$ in Fig. 2) to develop an upward bulge that results in the classic "pair of pants" picture of the merger $[3,4]$.

Introduction of this upward bulge into Fig. 2 leads to the model of an event horizon shown in Fig. 3, which otherwise has the same qualitative features. If we take into account the rotational symmetry suppressed in the figure, the light rays generating the horizon emerge from a spacelike surface $X$ that becomes asymptotically lightlike at the ring of cusps $C$ forming its boundary. The horizon generators emerge from the base $X$ in pairs, heading in the $\pm z$ directions. As we approach the caustic $C$ at the edge of $X$, the angle formed by each pair of generators closes up and gives the single generator emanating from that point of $C$.

It is now apparent how this model gives rise to toroidal black holes. Figure 3 shows a time slice of the horizon formed by its intersection with a (three-dimensional) Cauchy hypersurface $T_{1}$. It consists of two disjoint circles that form a torus, after factoring in the circles of

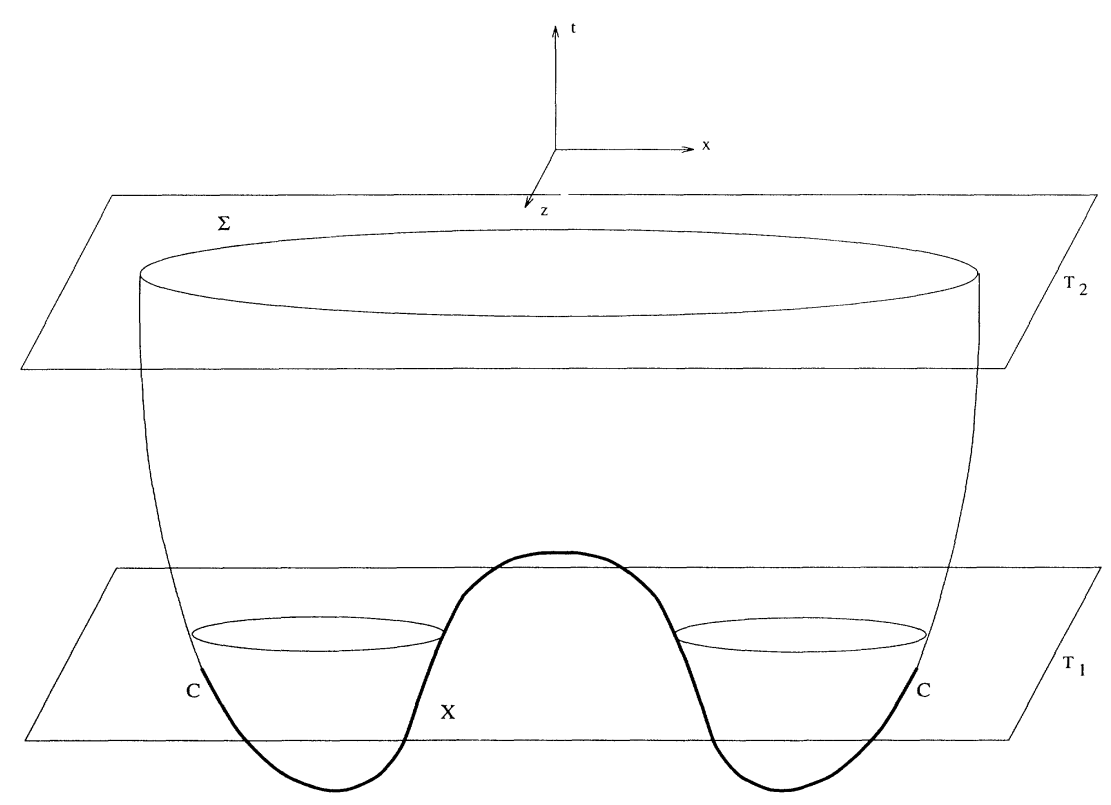

FIG. 3. The event horizon obtained on deforming Fig. 2 by the tidal effect of matter collapsing to a black hole. 
rotational symmetry suppressed in the figure. [Pairs of points with coordinates $(t, \pm x, z)$ in the figure lie on the same circle of symmetry.] Note that similar toroidal cross sections of $H$ can be found in the flat space case of Fig. 2, although this would require a curved time slice. Also note that a late time slice of the horizon, e.g., $T_{2}$ in Fig. 3, gives rise to a spherical black hole.

\section{THE GEOMETRY OF A TEMPORARILY TOROIDAL HORIZON}

We have used our numerical relativity code to simulate the collapse of rotating toroidal configurations of collisionless particles to Kerr black holes. We have found an interesting case in which the black hole event horizon initially develops as a toroid $[11,10]$. The initial configuration is based on a solution for a rotating toroidal cluster in stable equilibrium. The cluster has an outer circumferential radius of $R_{s} / M=4.5$. To get it to collapse we reduce the angular momentum of each particle by a factor of 0.5 , producing a nonequilibrium cluster with total angular momentum $J / M^{2}=0.70$. Spatial snapshots of the collapsing configuration, together with the location of the apparent and event horizons, are plotted in Figs. 6 and 7 of [10]. The toroidal horizon first forms entirely within the vacuum, between the origin and the inner edge of the toroidal cluster. It then expands to fill up the doughnut hole, becoming topologically spherical approximately when the outer edge of the horizon reaches the inner edge of the matter toroid.

The event horizon is found after the simulation is complete by propagating light rays backward in time from the surface of the final equilibrium black hole $[10,14]$. The spacetime reconstruction of the horizon is depicted in Figs. 4 and 5. They show some of the generating rays

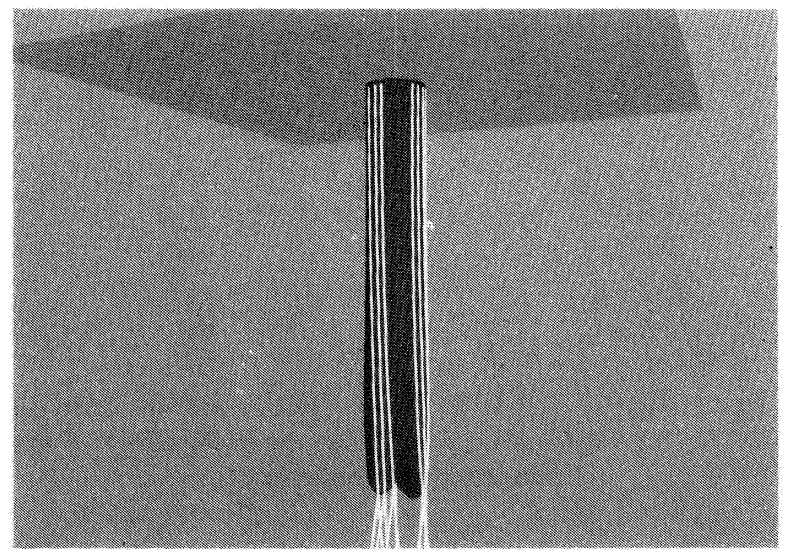

FIG. 4. Computational construction of the spacetime diagram for the collapse of the rotating toroid described in Sec. III. The time axis is vertical and the horizontal plane intersecting the axis is a spatial time slice. World lines of some of the light rays generating the horizon (shaded region) are shown. Clearly seen are the crossover points at which these light rays on the left enter the horizon, and the cusp formed by rays on the right at which the line of crossovers terminates. superimposed on the event horizon. We see that, tracing the rays backward in time, some of them leave the horizon at crossovers. For these rays the crossovers are the end points of the null generators of the horizon. The line of crossovers forms a spacelike curve. The figure is quite similar to the qualitative sketch shown in Fig. 3, and the similarity of the two figures gives us confidence that we understand the topological evolution of the horizon.

\section{CONCLUSION}

The computational simulation of a rotating black hole and its horizon structure, which we have presented here, is in complete accord with the various theorems regarding black hole topology. At times when the surface of the rotating black hole has a manifold structure, its surface topology is either toroidal or spherical, in agreement with the work of Gannon [6]. However, Gannon's theorem assumes a smooth black hole and it is unclear how it could be generalized to apply to the formation stage when new generators are being added. In our computational model, the toroidal black hole has a nonsmooth inner rim where new generators emerge from crossover points on $X$ (as well as a nonsmooth outer rim at the very early times before the caustic). Also, at the exceptional time at which the toroid pinches off, just prior to becoming spherical, the surface of the black hole is not even a (Hausdorff) manifold.

At late times, when equilibrium has been reached, the topology is spherical, in accord with the results of Hawking [5]. At early times, the topology defined by the Cauchy slicing is temporarily toroidal. However, the spacetime curve traced out by a point on the inner rim of the torus is spacelike, lying in $X$. Thus the "hole" in the torus closes up faster than the speed of light. Consequently, no causal signal can link through the torus and escape back to the exterior spacetime region to provide a violation of topological censorship, although the full implications that the theorem of Jacobson and Venkataramani [8] bears on this model deserve further study. Finally, at intermediate times to the future of $X$, when the

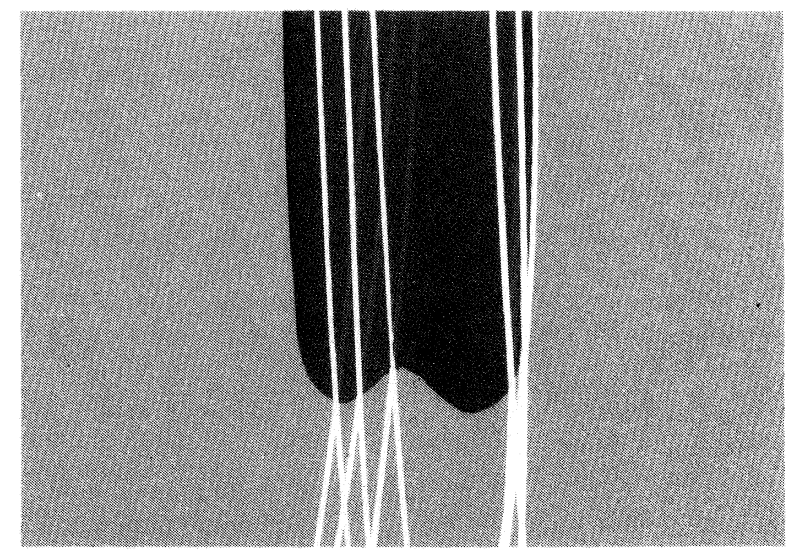

FIG. 5. Zooming in on the base of the horizon in Fig. 4, showing the crossovers and cusp. 
horizon has its full complement of generators, the rotating black hole has spherical topology, in agreement with the theorems of Browdy and Galloway [9].

\section{ACKNOWLEDGMENTS}

This research was supported by NSF Grand Challenge Grant No. PHY93-18152/ASC93-18152 (ARPA supple- mented). The work was also supported by NSF Grants Nos. PHY 92-08349 to the University of Pittsburgh and AST 91-19475 and PHY 94-08378 to Cornell University, as well as by NASA Grant No. NAG-2809 to Cornell. Computing resources were made available by the Pittsburgh Supercomputing Center and the Cornell Theory Center. We thank Ted Jacobson for interesting us in this problem.
[1] R. Penrose, Phys. Rev. Lett. 10, 66 (1963).

[2] R. Wald, General Relativity (University of Chicago Press, Chicago, 1984).

[3] S. W. Hawking and G. F. R. Ellis, The Large Scale Structure of Spacetime (Cambridge University Press, Cambridge, England, 1973).

[4] C. W. Misner, K. S. Thorne, and J. A. Wheeler, Gravitation (Freeman, San Francisco, 1973).

[5] S. W. Hawking, Commun. Math. Phys. 25, 152 (1972).

[6] D. Gannon, Gen. Relativ. Gravit. 7, 219 (1976).

[7] J. L. Friedmann, K. Schleich, and D. M. Witt, Phys. Rev. Lett. 71, 1486 (1993).

[8] T. Jacobson and S. Venkataramani, Class. Quantum Grav. 12, 1055 (1995).

[9] S. Browdy and G. J. Galloway, J. Math. Phys. 36, 4952 (1995); G. J. Galloway, in Contemporary Mathematics, edited by J. Beem and K. L. Duggal (Amer. Math. Soc., Providence, 1994), Vol. 170.

[10] S. A. Hughes, C. R. Keeton, P. Walker, K. Walsh, S. L. Shapiro, and S. A. Teukolsky, Phys. Rev. D 49, 4004 (1994).

[11] A. M. Abrahams, G. B. Cook, S. L. Shapiro, and S. A. Teukolsky, Phys. Rev. D 49, 5153 (1994).

[12] Spacetime properties of these caustics are described in H. Friedrich and J. M. Stewart, Proc. R. Soc. London A385, 345 (1983).

[13] R. A. Matzner, H. E. Seidel, S. L. Shapiro, L. Smarr, W.-M. Suen, S. A. Teukolsky, and J. Winicour, Science (to be published).

[14] P. Anninos, D. Bernstein, S. Brandt, J. Libson, J. Massó, E. Seidel, L. Smarr, W.-M. Suen, and P. Walker, Phys. Rev. Lett. 74, 630 (1995). 


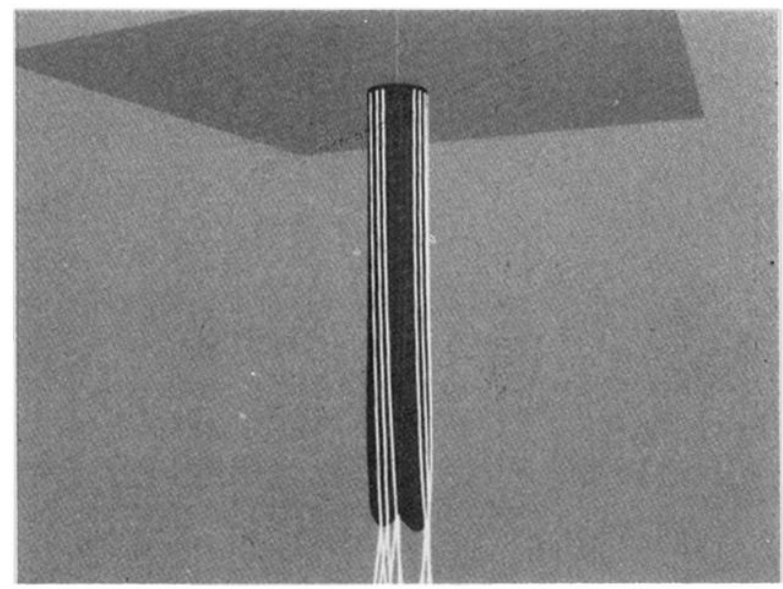

FIG. 4. Computational construction of the spacetime diagram for the collapse of the rotating toroid described in Sec. III. The time axis is vertical and the horizontal plane intersecting the axis is a spatial time slice. World lines of some of the light rays generating the horizon (shaded region) are shown. Clearly seen are the crossover points at which these light rays on the left enter the horizon, and the cusp formed by rays on the right at which the line of crossovers terminates. 


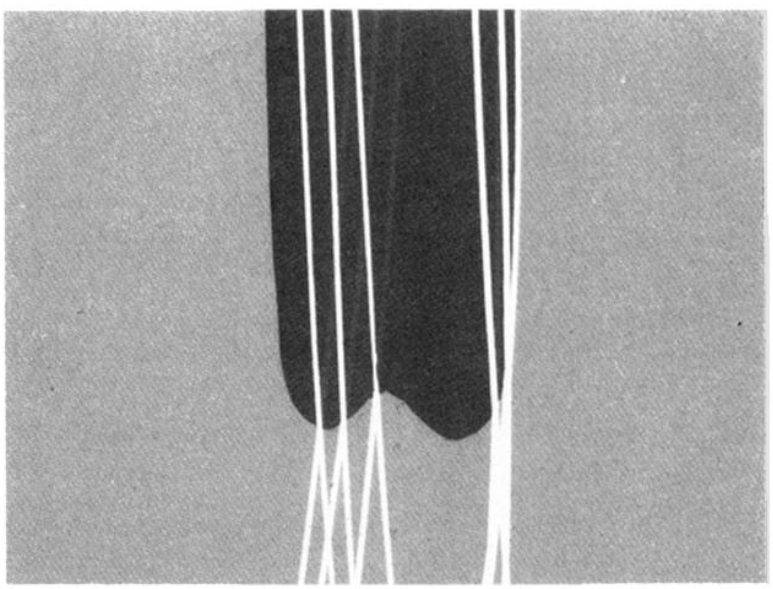

FIG. 5. Zooming in on the base of the horizon in Fig. 4, showing the crossovers and cusp. 